\title{
Review Article \\ Therapeutic Cancer Vaccines in Combination with Conventional Therapy
}

\author{
Mads Hald Andersen, ${ }^{1,2}$ Niels Junker, ${ }^{1,3}$ Eva Ellebaek, ${ }^{1,3}$ Inge Marie Svane,, 3 \\ and Per thor Straten ${ }^{1}$ \\ ${ }^{1}$ Center for Cancer Immune Therapy (CCIT), Department of Hematology, 54P4, Copenhagen University Hospital, \\ Herlev Ringvej 75, 2730 Herlev, Denmark \\ ${ }^{2}$ Department of Systems Biology, Technical University of Denmark, 2800 Kgs. Lyngby, Denmark \\ ${ }^{3}$ Department of Oncology, Copenhagen University Hospital, Herlev Ringvej 75, 2730 Herlev, Denmark
}

Correspondence should be addressed to Per thor Straten, pethst01@heh.regionh.dk

Received 5 February 2010; Revised 5 May 2010; Accepted 17 May 2010

Academic Editor: Zhengguo Xiao

Copyright (C) 2010 Mads Hald Andersen et al. This is an open access article distributed under the Creative Commons Attribution License, which permits unrestricted use, distribution, and reproduction in any medium, provided the original work is properly cited.

The clinical efficacy of most therapeutic vaccines against cancer has not yet met its promise. Data are emerging that strongly support the notion that combining immunotherapy with conventional therapies, for example, radiation and chemotherapy may improve efficacy. In particular combination with chemotherapy may lead to improved clinical efficacy by clearing suppressor cells, reboot of the immune system, by rendering tumor cells more susceptible to immune mediated killing, or by activation of cells of the immune system. In addition, a range of tumor antigens have been characterized to allow targeting of proteins coupled to intrinsic properties of cancer cells. For example, proteins associated with drug resistance can be targeted, and form ideal target structures for use in combination with chemotherapy for killing of surviving drug resistant cancer cells. Proteins associated with the malignant phenotype can be targeted to specifically target cancer cells, but proteins targeted by immunotherapy may also simultaneously target cancer cells as well as suppressive cells in the tumor stroma.

\section{Introduction}

Traditional cancer treatment modalities include surgery, radiation therapy, chemotherapy, and for some cancer types, hormone therapy. Although these treatment modalities are life extending for many patients, they are rarely curative for disseminated cancers. The use of cancer vaccines to induce a therapeutic antitumor immune response in the patient has huge potential to complement traditional cancer therapies in a nonoverlapping way. Therapeutic cancer vaccines are designed to recalibrate the existing host-tumor interaction, tipping the balance from tumor acceptance towards tumor control to the benefit of the cancer patient. Additionally, the highly specific character of the host immune response minimizes the risk for unattractive adverse events associated with most other cancer therapies in use today. Such vaccinations have been ongoing since early 1990s [1] based on the long awaited characterization of human tumor antigens recognized by patient $\mathrm{T}$ cells [2]. Encouraged by sporadic successes, mainly in small phase I trials $[3,4]$, a high number of trials have been ongoing worldwide, however so far the clinical efficacy as demonstrated in large phase III trials has in most cases be absent or marginal. Recently, however, the US Food and Drug Administration (FDA) approved an autologous cellular vaccine (Provenge $($ ) for the treatment of prostate cancer. The approval was based a clinical phase III trial including 512 patients with asymptomatic or minimally symptomatic, metastatic prostate cancer and was shown to increase overall survival (OS) by 4 months compared to placebo.

Initial attempts to improve vaccination induced biological and clinical responses focussed on the use of biological modifiers or adjuvants with the aim of increasing the magnitude of the response. To this end, immune stimulatory molecules such as interleukin-2 (IL-2) and granulocytemacrophage colony-stimulating factor (GM-CSF) have been 
used as adjuvants in many animal studies and vaccination trials. Over the past years it has been realized that the immune response is in fact comprised not only by responder cells and molecules, but also cells and molecules responsible for a counter-response $[5,6]$. In turn this has led to intensified research into the cells and molecules involved with suppressing immune responses, and ways to inhibit or delete the counter response [7]. In this regard, it has been realized that chemotherapy could be used to specifically target suppressor cells of the immune system [8] or to "reboot" the immune system for induction of anti-cancer responses [9]. Moreover, chemotherapy could lead to immunogenic death of tumor cells [10], depending on the drug, dose, and administration.

Concerning the characterization of target epitopes recognized by $\mathrm{T}$ cells, the past decades has been characterized by an increased focus on proteins that functionally link to the malignant phenotype, for example, to metastasize and avoid apoptosis [11-16]. Importantly, some of the tumor associated antigens that comprise antigenic peptides are functionally related to resistance to conventional therapy, setting the stage for specifically targeting of cells that escape conventional therapy [17-21]. Last but not least, the tumor supportive role of cells in the stroma have encouraged the characterization of antigens which are expressed not alone by cancer cells but also or exclusively by cells in the tumor stroma [21-25].

Therapeutic strategies based on the above findings are likely to improve the clinical efficacy of therapeutic vaccinations against cancer. Nonetheless, combinations of chemotherapy-may be even with several drugs in combination-with immunological strategies, are complex to evaluate, and demand for careful design of clinical trials as well as development of new ways to evaluate dose and schedule combinations rationally [26]. The perspective, however, is future treatments that combine immunological treatment with conventional therapy leading to increased clinical efficacy to the benefit of the patient.

\section{Combination with Chemotherapy Immunological Reboot, and Immunological Cell Death}

Even few years back, the idea of combining chemotherapy with active immune therapy was unheard of. The dogma stated that chemotherapy would jeopardize any immune responses, in part due to the fact that dividing immune cells are vulnerable to chemotherapeutic drugs, and as a consequence concurrent active immune therapy with chemotherapy would be pointless. Nonetheless, the concept of combining immune therapy with sequential or even concurrent chemotherapy has lately gained much interest, for several reasons. First, a number of surprising findings suggested that combining immuno- and chemotherapy could lead to better responses in advanced cancer patients [27]. To this end, data from several studies suggest that clinical response to chemotherapy is improved if preceded by immunotherapy [28-31]. A possible explanation for this phenomenon is that cancer cell death by chemotherapy will lead to presentation of antigens that may further activate a treatment-induced $\mathrm{T}$ cell response leading to increased killing of cancer cells by $\mathrm{T}$ cells.

Obviously, not only cancer cells are inflicted by systemic chemotherapy but also cells of the tumor stroma and the immune system are influenced and as a consequence any improved reactivity may rely on effects imposed on the stromal cells, immune system, or on cancer cells-and probably all may play a role. In general terms, the influence of chemotherapy may have an impact on normal cells of the host; for example, cells of the immune system as well as an impact on cancer cells. These issues are discussed in some more detail below.

\subsection{Chemotherapy in Relation to Normal Cells; Depletion} of Suppressor Cells. Previously, there has been tremendous focus on the use of molecules or cells that would work in direction of a more powerful activation of the immune system in any given immunotherapy, however, the recent acknowledgement that counter-active cells are at play during immune responses has led to a focus on suppressor mechanisms. There seem to be at least two cell types involved as key players in cancer, myeloid derived suppressor cells (MDSC) [6], and regulatory $\mathrm{T}$ cells (Treg) [32]. MDSC are suppressor cells of the innate immune system capable of inhibiting both innate and adaptive immune responses [33]. Similarly, regulatory $\mathrm{T}$ cells of the adaptive immune system are capable of suppressing immune responses $[5,34]$. Both cell types may be found in high frequencies in cancer patients-in blood but also at the tumor site, evidence is now emerging that both cell types are clinically relevant and predictive for patient survival $[6,35,36]$. Obviously, these suppressive cells play important roles in controlling and adjusting immune responses in general and are not in any way restricted to cancer patients. Hence, the existence of these populations of suppressive cells underscores the self-limiting nature of the immune system, characterized by dual actions, that is, the capacity to kill and destroy upon antigen recognition as well as the capacity to promote repair after antigen clearance, removal of dead cells and microbes, and the subsequent construction of new vessels and down expression of danger signals. This delicate balance is not only governed by presence or absence of antigen, but by a variety of cellular interactions and soluble factors. Thus, any immune response is composed by active as well as counteractive cells, and the mechanisms that control the immune response from initiation to full completion and repair are poorly understood.

It is now generally accepted that there is a link between inflammation and cancer-in turn leading to the notion that a tumor represents a group of cells selected for the capacity to induce immunological repair instead of clearance. Moreover, the intimate relation between inflammation and cancer underscores that the interplay between cells of the immune system and cancer cells are ongoing from initiation of oncogenesis and thus that suppressive or repair mechanisms are an intrinsic phenomenon of cancer [37]. Although data accumulating over the past few years have revealed much 
new insight into the counter-active cells of the immune system, we may just have seen the tip of the iceberg. We recently identified regulatory $\mathrm{CD} 8 \mathrm{~T}$ cells present in high frequencies in cancer patients, and capable of suppressing cytokine production, proliferation, and cytotoxic activity of other $\mathrm{T}$ cells with a hitherto unheard efficacy [38]. Reaching frequencies of $0.2 \%$ among peripheral blood lymphocytes (PBL) these CD8 $\mathrm{T}$ cells are specific for a single peptide from the protein heme oxygenase 1 (HO-1). Interestingly, this protein is expressed by tumor cells as well as during wound healing [39] and is a key molecule in local immune suppression [40], thus coupling the action of the novel CD8 T-cell population with the overall function of the protein recognized. The characterization of these antigen-specific suppressive CD8 $\mathrm{T}$ cells in cancer patients underscores that there are pros and cons of the immune system in relation to the interactions between cells of the immune system and cancer cells.

Thus, Treg, be it CD4 or CD8 cells seem to play a role for suppression of anticancer immune responses, but other cells in the tumor stroma as well as cancer cells themselves may also possess suppressive functions. Normal cells in the stroma and a fraction of cancer cells may express indoleamine 2,3-dioxygenase (IDO) [41, 42]. IDO may suppress $\mathrm{T}$-cell responses directly by tryptophan deprivation in the microenvironment, and dendritic cells (DCs) may also express IDO leading to induction of Treg [43]. In turn, Treg may in fact induce IDO expression in DC further linking the suppressive network [44]. The drug 1-MT that inhibit IDO activity is in clinical testing [45], and may represent a suitable conditioning or combination partner to immunotherapy. Interestingly, we recently revealed that IDO is a target for specific $\mathrm{T}$ cells in cancer patients, and that such specific cells are capable of killing IDO-expressing tumor cells and also DC provided they express IDO [24]. Thus, the induction of a response against IDO could diminish the suppressive effect of IDO and thereby "unleash" a powerful response against the full spectra of tumor antigens expressed by the cancer cells. Strikingly, we have used 1-MT added to peptide stimulation cultures and shown that in some cultures there is a marked increase in INF- $\gamma$ secretion measured by EliSpot, suggesting that PBMC cultures that comprise high frequencies of IDOpositive cells can be suppressive to $\mathrm{T}$ cells-a suppression that can be unleashed by 1-MT [46].

Steps to clear Treg cells prior to vaccination by a single administration of chemotherapy, for example, cyclophosphamide (CTX) have been attempted and have been shown to increase induced immune responses in murine [47] as well as human studies $[48,49]$. However, a single clearance does not appreciate the continuous development of Treg [50], and other suppressive cells. Continued administration of low dose CTX was recently shown to be able to selectively clear Treg and would therefore be far more appealing [8]. However, we recently conducted a clinical vaccination trial in melanoma patients using the described low dose CTX without being able to induce Treg clearance or even decrease in Treg frequency (manuscript in preparation). Also taxanes which are widely used in the clinic have been studied for impact on cells of the immune system in particular Treg
$[51,52]$. Data from such studies suggest that both paclitaxel and docetaxel may impair the frequency of Treg either alone [51] or in combination with carboplatin [53] or CTX [54]. It could be speculated that the impact of chemotherapy on Treg depends on the subtype of the regulatory cell. To this end, it has been shown that induced Treg are rapidly dividing [55], and such cells could be more vulnerable to chemotherapy.

Concerning manipulations with MDSC this has been tested in murine models, where Gemcitabine were shown to selectively eliminate GR1 positive MDSC [56]. Similarly, it has been found that unresponsive tumor infiltrating lymphocytes (TIL) of prostate cancer can gain responsiveness by blockage of arginase 1 (ARG) known to be expressed by MDSC [57]. Cyclooxgenase 2 (COX-2) seems to play a role for induction of MDSC, and administration of COX-2 inhibitors have been shown to decrease MDSC development $[58,59]$. We are currently running a clinical trial in melanoma patients in which DC vaccination is combined with concurrent administration of the COX2 inhibitor Celebra (http://www.clinicaltrial.gov/). Whereas most attempts to manipulate suppressive cells like MDSC focus on lowering the frequency of the cells, a recent report demonstrated blockage of the suppressive function of MDSC by synthetic triterpenoid whereas the frequency of the cells was unchanged [60]. A potential difficulty concerning selective clearing or blockage of function of MDSC using chemotherapy is that these cells seem to possess different phenotypes in different cancers [6], and have different ways of actions $[61,62]$ which could be coupled to highly different properties of the cells in response to chemotherapeutic drugs.

Whether single individual drugs may synergistically work with, for example, therapeutic vaccination by selective clearing or decreasing the activity of cells with suppressive function remains so far elusive, and certainly we are by no means close to having defined optimal dosage, schedule, and so forth, for such combinations. Moreover, many cellular approaches are based on the use of allogeneic tumor cell lines transfected with vectors encoding immune stimulatory molecules, which obviously leads to a next level of complexity since the beneficial combination with chemotherapy may depend on the added stimulatory molecule. To this end, two recent studies in which allogeneic, GM-CSF-secreting tumor vaccines were administered to cancer patients in combination with CTX (and doxorubicin) suggested higher efficacy in the CTX group $[26,63]$. These highly complex combinations will benefit from designs that are geared to identify the most clinically relevant combination of the interacting drugs [26].

\subsection{Chemotherapy in Relation to Normal Cells; Conditioning prior to Adoptive Cell Transfer (ACT). As mentioned, it is still questionable whether selective clearing of immune cells with suppressive function is possible using chemotherapy; however, chemotherapy could also be beneficially used to "reboot" the immune system prior to initiation of immune therapy against cancer. For example, the efficacy of ACT using in vitro expanded TIL in melanoma patients seems to depend on prior conditioning using chemotherapy}


and/or whole body irradiation therapy [64]. The biological background for the requirement of conditioning remains unknown but is probably related to creating space thereby enabling homeostatic cell division of transferred cells, and clearing of suppressive cells in the patient [9]. Detailed insight into the mechanisms that influence the success of TIL in ACT could potentially open the avenue for development of an ACT regimen that are associated with fewer and less serious side effect.

The induction of clinically relevant responses by ACT using lympho depleting procedures and TIL transfer could also in part be related to the broad spectra of reactivity comprised in these TIL cultures. In accordance, we have studied such TIL cultures from melanoma and head and neck cancer patients for reactivity against a panel of tumor antigens using EliSpot and tetramer analyses, and in general many of these cultures comprise a large number of Tcell specificities (paper in preparation). T-cell survival and expansion within the host depends on the availability of growth-promoting cytokines and regular encounters with cognate antigen. Under ideal conditions small numbers of infused $\mathrm{T}$ cells can undergo massive expansion in vivo [65]. However, this level of in vivo expansion may not occur following the adoptive transfer of tumor antigen-specific $\mathrm{T}$ cells owing to the poor immunogenicity of the tumor. Hence, an alternative approach is to combine adoptive transfer of $\mathrm{T}$ cells with vaccination to facilitate expansion and maintenance of $\mathrm{T}$ cells. The combination of ACT of either in vitro expanded specific $\mathrm{T}$ cells or gene-modified $\mathrm{T}$ cells [66] with vaccination [67] might provide synergism between the two treatment regimens.

2.3. Chemotherapy in Relation to Normal Cells; Activation of Cells of the Immune System. Chemotherapy may also influence cells of the immune system to more potent activity. To this end, it has been shown that gemcitabine treatment lead to increased efficacy of immune therapy in the absence of any direct effect on cancer cells [68]. As mentioned above CTX may have a role in the clearing of Treg and is used together with fludarabin as conditioning prior to ACT. Using CTX alone, it was recently shown in a murine model that the myelosuppressive action may lead to subsequent rebound DC generation with increased capacity to secrete IL-12 [69]. With a more direct action on DC, vinblastine has been shown to induce DC maturation [70], and functionally these DCs were superior to untreated DC in inducing CD8 T-cell responses [71]. Using noncytotoxic concentrations, it was recently shown that several clinically relevant drugs (paclitaxel, doxorubicin, mitomycin $\mathrm{C}$, and methotrexate) increase antigen presentation in an autocrine IL-12-dependent manner [72]. Thus, these data suggest that provided careful examination of the dose and schedule, it might be possible to harness the dual actions of chemotherapy to kill some cells and activate others.

2.4. Chemotherapy; Impact on Cancer Cells. Chemotherapy may also induce an antitumor immune response by direct influence on the tumor cells. To this end, a panel of chemotherapeutic agents was screened for inducing immunological cell death, each drug was studied functionally for the ability of chemotherapy-killed tumor cells to induce protective immunity upon immunization [73]. The data demonstrated that anthracyclin-treated tumor cells are particularly effective in eliciting an anticancer immune response, and further that the mechanism of the immunogenicity of antracyclin-induced cell death was the rapid preapoptotic translocation of calreticulin to the cell surface [73]. This surface exposure of calreticulin endows cancer cells with an "eat me" signal to dendritic cells, in turn leading to immunogenic uptake of tumor antigens and activation of tumor-specific T-cell responses [73]. Thus, to successfully combine chemotherapy with immune therapy careful selection of the chemotherapeutic agent is required since not all agents induce immunological death [74].

Another key denominator of immunogenic cell death is represented by high-mobility group box 1 (HMGB1). Release of HMGB-1 from dying tumor cells leads to activation of toll-like receptors (TLRs) (2 and 4) and subsequent immune activation [75]. In this respect, HMGB1 localization in the cytosol is associated with autophagy and cellular escape from apoptosis, in turn conferring resistance to several therapies including immunotherapy [74]. Also heat shock proteins (HSPs) which are upregulated upon specific stress may act as danger signals and be expressed on the cell surface as "eat me" signals to DC [76]. Interestingly, it has been shown that treatment of myeloma cells with the proteasome inhibitor bortezomib leads to surface expression of HSP90 on the cell surface [77]. A vaccination trial in which bortezomib is combined with peptide vaccination targeting the regulators of apoptosis proteins (RAPs) Bcl-2, Mcl-1, and $\mathrm{Bcl}-\mathrm{Xl}$ [21] was recently initiated at our institution (www.clinicaltrial.gov).

Beyond inducing immunogenic tumor cell death, chemotherapy may render cancer cells more susceptible to killing by CTL. Thus, 5-fluorouracil (5-FU), CPT-11, or cisplatin (CDDP) were all shown to increase the sensibility of the SW480 colon cancer cell line to killing by T cells [78], and similar data have been found for renal cell carcinoma cells treated with adriamycin [79]. In both cases the enhanced lytic sensitivity was at least in part due to upregulation of costimulatory molecules on cancer cells, for example, LFA-3 and ICAM-1. More recently, Ramakrishnan and colleagues showed in a murine model that paclitaxel, cisplatin, and doxorubicin all sensitize tumor cells to more efficient killing by CTL [80]. In this system, however, the responsible mechanism on murine as well and human cancer cells went via upregulation of the mannose-6-phosphate (M6P) receptor on cancer cells which is required for granzyme $\mathrm{B}$ associated killing [81]. Importantly, CTL established upon administration of chemotherapy was capable of off-target killing of neighboring cells at the tumor site even in the absence of antigen expression.

The concept of using chemotherapy as an immune adjuvant is rapidly expanding. Obviously, future testing in clinical trials will benefit from basic research into the mechanism(s) of action; how do the cells die and which pathways are activated for immune activation $[82,83]$. This 
will set the stage for initiation of hypothesis driven clinical trials combining chemotherapy and immune therapy and form the basis for more rational biological monitoring.

\section{Chemotherapy Resistance and Immune Targeting}

Chemotherapeutic agents can induce a series of cellular responses that impact on tumor cell proliferation and survival. Perhaps the best studied of these cellular responses is apoptosis, a physiological cell death program that controls normal cell numbers during development and disease. A large number of various drugs in clinical use kill tumor cells by activation of common apoptotic pathways. Hence, most cytotoxic anticancer drugs, for example, microtubule binding drugs, DNA-damaging agents, and nucleosides induce apoptosis of malignant cells.

Many drugs are capable of inducing clinical response in the patients with metastatic cancer, however, in most cases the therapy is not curative due to selection of drugresistant cancer cells. A frustrating property of such acquired resistance of cancer cells is that chemotherapy resistance may lead to cross-resistance to other drugs with different mechanisms of action [84]. Drug resistance is a major limiting factor for the effectiveness of chemotherapy in the treatment of disseminated cancer [85]. Cancer-associated defects in apoptosis play a vital role in resistance to chemotherapy and radiotherapy [86]. An important reason for this impaired apoptosis is overexpression of RAP [87], that is, the T-cell antigens survivin and proteins of the Bcl-2 family. Other mechanisms of drug resistance are exemplified by the tumor antigen CYP1B1 which may inactivate cytotoxic or cytostatic drugs thereby influencing the clinical outcome of therapy [88], and ATP transporters which act by transporting drugs out of the cell [89].

The mechanisms of drug resistance mentioned above are associated with expression of proteins that have been shown to be targets for $\mathrm{T}$ cell responses [17-21, 90-95]. Consequently, the combination of immunotherapy targeting these antigens with conventional chemotherapy appears to be particularly appealing. In such a setting, conventional therapy would kill the majority of the cancer cells, leaving only cells that express high levels of target antigens. Such high-expressers would be particularly vulnerable to killing by vaccination-induced $\mathrm{T}$ cells. Thus, the synergy of these measures could potentially give a more effective treatment than the added effect of either regimen alone, thereby strengthening the already described synergistic effect of anticancer vaccines and chemotherapy. Furthermore, it should be noted that chemotherapy only has an effect on dividing cells, whereas, for example, surviving-specific $\mathrm{T}$ cells in addition are able to kill resting tumor cells.

\section{Radiation Therapy in Combination with Vaccination}

Irradiation leads to immunogenic death [73]. Moreover, also sublethal irradiation may render cancer cells more vulnerable to killing by $\mathrm{T}$ cells, implying that even surviving irradiated cancer cells may still facilitate more efficient responses. Total body irradiation (TBI) is already being used by Dudley and colleagues as part of a conditioning regimen prior to ACT [9], indicating additive with more intensified TBI. Interestingly, a well-known phenomenon related to local radiation therapy is a bystander effect extending to distant untreated metastatic sites - an effect potentially mediated by the immune system [96]. Probably radiation could be pursued more conceptually in combination with vaccination as well.

\section{Specific or Concurrent Targeting of Stroma Cells}

One of the inherent properties of cancer cells is genetic instability which in turn allows cancer cells to "escape" during therapy. Obviously, the "active-imposing" term "escape" is in fact a selection process in which cells carrying an advantageous genetic, epi-genetic, and miRNA signature are given a survival and/or growth advantage. As already mentioned, this is a crucial problem concerning chemotherapy, but also for the success of immune therapy this poses a problem. In this regard, antigen loss [97] or HLA loss [98] has been described during immune therapy, and the background for this lies in the heterogeneity of cancer cells in turn enabling the presence of cells with escape properties. Conversely, stroma cells are genetically stable and possess limited proliferative capacity compared to cancer cells, implying that the risk of HLA-loss, antigen-loss, or antigen processing-loss is exceedingly low. Some antigens are expressed not only by tumor cells but also by cells in tumor stroma. This applies to survivin, and several other RAP.

Angiogenesis represents an important step in tumor development [99], and since antiangiogenic therapy targets the tumor vasculature and prevents tumor growth beyond micro-metastases, combination of antiangiogenic therapy and tumor-specific immunotherapy could lead to a synergistic effect. Active immunotherapy targeting endothelial products like vascular endothelial growth factor receptor (VEGFR)-2 protein can delay tumor progression [22], and it has been shown that vaccination with peptides derived from VEGFR-1 inhibits tumor growth in mice. This was associated with suppression of tumor angiogenesis in the absence of adverse effects [100]. In addition, similar to survivin, Bcl-2 and Mcl-1 are highly expressed in endothelial cells during tumor angiogenesis [101]. Thus, the targeting of survivin, Bcl-2, or Mcl-1 in a vaccination setting would beyond tumor cells also target endothelial cells and thus tumorangiogenesis. Indeed this was shown in a mouse model; vaccination-induced survivin-specific $\mathrm{T}$ cells mediated the eradication of lung tumor metastases and the concurrent suppression of angiogenesis at the tumor site [102]. The efficacy of treatment did not inflict on wound healing or fertility of the mice.

A primary concern of immunizing against angiogenesisassociated proteins is a potential risk of interference with normal angiogenesis, especially if the effect is sustained. So far no vaccination associated toxicity was observed when late 
stage melanoma patients were vaccinated with survivin in a compassionate use setting despite the fact that strong CTL responses were introduced in all patients [103]. Nevertheless, it is clear that even extensive phase I/II trials are not geared for analyses of potential side effects presenting several years after termination of the trial, and these issues demand further attention.

Certainly several targets are available for targeting of tumor stroma in particular for concurrent targeting of tumor cells and stroma cells. As already mentioned, IDO are expressed by fractions of cancer cells in vivo [42], but DCs, MDSC, and other immune cells in the tumor stroma may also be IDO positive, and characterized by the same targeting advantages as, for example, endothelial cells. To this end, it has been shown that regulatory $\mathrm{T}$ cells can be targeted by immune responses to peptides derived from Foxp3 [25].

\section{Multiepitope Strategies and Additional Immunotherapy}

Many current immunological strategies to combat cancer are already focusing on combining different cells and molecules to increase responses, for example, vaccination combined with the addition of cytokines or other immune modulating agents. However, so far most peptide-based vaccination trials have targeted only a single antigen and with the aim to increase efficacy an exciting strategy would be to cotarget biologically connected proteins, for example, RAP, in a multiepitope setting [104]. A number of different RAP have been described as T-cell antigens in a variety of cancers $[21,90,105,106]$. Since coexpression of Bcl-2 family proteins is a frequent event [87], simultaneous targeting of Bcl-2 proteins may be more efficient than targeting one molecule alone.

Importantly, drug resistance is casually linked to over expression of RAP, for example, survivin and Bcl-2 [107, 108]. For combination therapies, vaccination against these molecules seems to be ideally suited for targeting of chemoresistant cancer cells upon conventional therapy, and thereby possibly prevents relapse of disease [109]. Underscoring this notion, the coexpression of survivin and $\mathrm{Bcl}-2$ is associated with poor prognosis in breast cancer [110], implying that expression of more than one RAP is functionally significant at least in some indications. To this end, targeting both the Bcl-2 family proteins and survivin would be particularly attractive since they act in different apoptosis pathways, and the targeting of both to kill resistant cells upon chemotherapy would supposedly increase the chances of success. Combined with adjuvant chemotherapy after primary surgery, adjuvant vaccination in high risk cancer patients would be suited for a vaccination that specifically targets not only apoptosis pathways but also proteins associated with metastatic behavior, for example, RhoC $[16,111]$ or heparanase [15].

\section{Conclusions}

The immune system has a unique capacity to specifically recognize and kill cancer cells while leaving normal cells unharmed. As a consequence, to harness the immune system in therapeutic vaccinations against cancer is a very promising approach for the therapy of disseminated cancer. So far, however, the clinical impact of vaccination has been limited. Over the past few years, New insight has been achieved concerning the main suppressive mechanisms that hamper induction of more powerful immune responses, and also revealed new knowledge as to how suppressive cells and molecules could potentially be cleared or inhibited, for example, in combination with chemotherapy. Similarly, the immunogenicity of cancer cells has been shown to depend on the death process induced by the specific drug, thus some drugs are more prone to act synergistically with vaccination than others. Added to the above, cancer cells treated with chemotherapy may be rendered more vulnerable to killing by $\mathrm{T}$ cells, only adding to the potential of improving the efficacy of vaccination when combined with chemotherapy. Moreover, antigens may be selected and combined to target various traits of cancer cells and/or target stroma cells in the suppressive tumor environment. The available experimental data should form the basis for initiation of carefully planned hypothesis driven clinical trials that coupled with stringent and robust biological and clinical monitoring will be able to firmly demonstrate the most effective combinations.

\section{References}

[1] M. Marchand, P. Weynants, E. Rankin et al., "Tumor regression responses in melanoma patients treated with a peptide encoded by gene MAGE-3," International Journal of Cancer, vol. 63, no. 6, pp. 883-885, 1995.

[2] P. van der Bruggen, C. Traversari, P. Chomez et al., "A gene encoding an antigen recognized by cytolytic $\mathrm{T}$ lymphocytes on a human melanoma," Science, vol. 254, no. 5038, pp. 1643-1647, 1991.

[3] F. O. Nestle, S. Alijagic, M. Gilliet et al., "Vaccination of melanoma patients with peptide- or tumor lysate-pulsed dendritic cells," Nature Medicine, vol. 4, no. 3, pp. 328-332, 1998.

[4] S. A. Rosenberg, J. C. Yang, D. J. Schwartzentruber et al., "Immunologic and therapeutic evaluation of a synthetic peptide vaccine for the treatment of patients with metastatic melanoma," Nature Medicine, vol. 4, no. 3, pp. 321-327, 1998.

[5] S. Sakaguchi, K. Wing, Y. Onishi, P. Prieto-Martin, and T. Yamaguchi, "Regulatory T cells: how do they suppress immune responses?" International Immunology, vol. 21, no. 10, pp. 1105-1111, 2009.

[6] S. Ostrand-Rosenberg and P. Sinha, "Myeloid-derived suppressor cells: linking inflammation and cancer," Journal of immunology, vol. 182, no. 8, pp. 4499-4506, 2009.

[7] G. C. Prendergast and E. M. Jaffee, "Cancer immunologists and cancer biologists: why we didn't talk then but need to now," Cancer Research, vol. 67, no. 8, pp. 3500-3504, 2007.

[8] F. Ghiringhelli, C. Menard, P. E. Puig et al., "Metronomic cyclophosphamide regimen selectively depletes $\mathrm{CD} 4^{+} \mathrm{CD} 25^{+}$ regulatory $\mathrm{T}$ cells and restores $\mathrm{T}$ and $\mathrm{NK}$ effector functions in end stage cancer patients," Cancer Immunology, Immunotherapy, vol. 56, no. 5, pp. 641-648, 2007.

[9] M. E. Dudley, J. C. Yang, R. Sherry et al., "Adoptive cell therapy for patients with metastatic melanoma: evaluation 
of intensive myeloablative chemoradiation preparative regimens," Journal of Clinical Oncology, vol. 26, no. 32, pp. 5233 5239, 2008.

[10] R. G. van der Most, A. J. Currie, B. W. S. Robinson, and R. A. Lake, "Decoding dangerous death: how cytotoxic chemotherapy invokes inflammation, immunity or nothing at all," Cell Death and Differentiation, vol. 15, no. 1, pp. 13$20,2008$.

[11] A. W. Silk and O. J. Finn, "Cancer vaccines: a promising cancer therapy against all odds," Future Oncology, vol. 3, no. 3, pp. 299-306, 2007.

[12] P. A. Würtzen, L. $\varnothing$. Pedersen, H. S. Poulsen, and M. H. Claesson, "Specific killing of P53 mutated tumor cell lines by a cross-reactive human HLA-A2-restricted P53-specific CTL line," International Journal of Cancer, vol. 93, no. 6, pp. 855861, 2001.

[13] M. P. M. Vierboom, H. W. Nijman, R. Offringa et al., "Tumor eradication by wild-type p53-specific cytotoxic T lymphocytes," Journal of Experimental Medicine, vol. 186, no. 5, pp. 695-704, 1997.

[14] B. Maecker, M. S. von Bergwelt-Baildon, K. S. Anderson et al., "Rare naturally occurring immune responses to three epitopes from the widely expressed tumour antigens hTERT and CYP1B1 in multiple myeloma patients," Clinical and Experimental Immunology, vol. 141, no. 3, pp. 558-562, 2005.

[15] N. Sommerfeldt, P. Beckhove, Y. Ge et al., "Heparanase: a new metastasis-associated antigen recognized in breast cancer patients by spontaneously induced memory T lymphocytes," Cancer Research, vol. 66, no. 15, pp. 7716-7723, 2006.

[16] L. Wenandy, R. B. Sørensen, I. M. Svane, P. T. Straten, and M. H. Andersen, "RhoC a new target for therapeutic vaccination against metastatic cancer," Cancer Immunology, Immunotherapy, vol. 57, no. 12, pp. 1871-1878, 2008.

[17] A. Meier, S. Reker, I. M. Svane et al., "Spontaneous T-cell responses against peptides derived from the Taxol resistanceassociated gene-3 (TRAG-3) protein in cancer patients," Cancer Immunology, Immunotherapy, vol. 54, no. 3, pp. 219228, 2005.

[18] P. Kvistborg, S. R. Hadrup, I. M. Svane, M. H. Andersen, and P. T. Straten, "Characterization of a single peptide derived from cytochrome P450 1B1 that elicits spontaneous human leukocyte antigen (HLA)-A1 as well as HLA-B35 restricted CD8 T-cell responses in cancer patients," Human Immunology, vol. 69, no. 4-5, pp. 266-272, 2008.

[19] B. Maecker, D. H. Sherr, R. H. Vonderheide et al., "The shared tumor-associated antigen cytochrome P450 1B1 is recognized by specific cytotoxic T cells," Blood, vol. 102, no. 9, pp. 3287-3294, 2003.

[20] A. Yamada, K. Kawano, M. Koga, T. Matsumoto, and K. Itoh, "Multidrug resistance-associated protein 3 is a tumor rejection antigen recognized by HLA-A2402-restricted cytotoxic T lymphocytes," Cancer Research, vol. 61, no. 17, pp. 64596466, 2001.

[21] M. H. Andersen, J. C. Becker, and P. T. Straten, "Regulators of apoptosis: suitable targets for immune therapy of cancer," Nature Reviews Drug Discovery, vol. 4, no. 5, pp. 399-409, 2005.

[22] A. G. Niethammer, R. Xiang, J. C. Becker, et al., "The vascular endothelial growth factor receptor 2: a self-antigen recognized by cytotoxic T cells mediating tumor-protective immunity," Nature Medicine, vol. 8, pp. 1369-1375, 2002.

[23] Y. Luo, H. Zhou, J. Krueger et al., "Targeting tumorassociated macrophages as a novel strategy against breast cancer," Journal of Clinical Investigation, vol. 116, no. 8, pp. 2132-2141, 2006.

[24] R. B. Søorensen, L. Berge-Hansen, N. Junker et al., "The immune system strikes back: cellular immune responses against indoleamine 2,3-dioxygenase," PLoS One, vol. 4, no. 9, Article ID e6910, 2009.

[25] S. Nair, D. Boczkowski, M. Fassnacht, D. Pisetsky, and E. Gilboa, "Vaccination against the forkhead family transcription factor Foxp3 enhances tumor immunity," Cancer Research, vol. 67, no. 1, pp. 371-380, 2007.

[26] L. A. Emens, J. M. Asquith, J. M. Leatherman, et al., "Timed sequential treatment with cyclophosphamide, doxorubicin, and an allogeneic granulocyte-macrophage colony-stimulating factor-secreting breast tumor vaccine: a chemotherapy dose-ranging factorial study of safety and immune activation," Journal of Clinical Oncology, vol. 27, no. 35, pp. 5911-5918, 2009.

[27] R. Ramakrishnan, S. Antonia, and D. I. Gabrilovich, "Combined modality immunotherapy and chemotherapy: a new perspective," Cancer Immunology, Immunotherapy, vol. 57, no. 10, pp. 1523-1529, 2008.

[28] C. J. Wheeler, A. Das, G. Liu, J. S. Yu, and K. L. Black, "Clinical responsiveness of glioblastoma multiforme to chemotherapy after vaccination," Clinical Cancer Research, vol. 10, no. 16, pp. 5316-5326, 2004.

[29] P. M. Arlen, J. L. Gulley, C. Parker et al., "A randomized phase II study of concurrent docetaxel plus vaccine versus vaccine alone in metastatic androgen-independent prostate cancer," Clinical Cancer Research, vol. 12, no. 4, pp. 1260-1269, 2006.

[30] S. J. Antonia, N. Mirza, I. Fricke et al., "Combination of p53 cancer vaccine with chemotherapy in patients with extensive stage small cell lung cancer," Clinical Cancer Research, vol. 12, no. 3, pp. 878-887, 2006.

[31] J. G. Gribben, D. P. Ryan, R. Boyajian et al., "Unexpected association between induction of immunity to the universal tumor antigen CYP1B1 and response to next therapy," Clinical Cancer Research, vol. 11, no. 12, pp. 4430-4436, 2005.

[32] S. Sakaguchi, "Naturally arising Foxp3-expressing CD $25^{+}$ $\mathrm{CD}^{+}$regulatory $\mathrm{T}$ cells in immunological tolerance to self and non-self," Nature Immunology, vol. 6, no. 4, pp. 345-352, 2005.

[33] S. Ostrand-Rosenberg, “ Myeloid-derived suppressor cells: more mechanisms for inhibiting antitumor immunity," Cancer Immunology and Immunotherapy. In press.

[34] D. Mougiakakos, A. Choudhury, A. Lladser, R. Kiessling, and C. C. Johansson, "Regulatory T cells in cancer," Advances in Cancer Research, vol. 107, pp. 57-117, 2010.

[35] C. Ménétrier-Caux, M. Gobert, and C. Caux, "Differences in tumor regulatory T-cell localization and activation status impact patient outcome," Cancer Research, vol. 69, no. 20, pp. 7895-7898, 2009.

[36] H. Schmidt, S. Suciu, C. J. A. Punt et al., "Pretreatment levels of peripheral neutrophils and leukocytes as independent predictors of overall survival in patients with American Joint Committee on Cancer stage IV melanoma: results of the EORTC 18951 biochemotherapy trial," Journal of Clinical Oncology, vol. 25, no. 12, pp. 1562-1569, 2007.

[37] K. E. de Visser, A. Eichten, and L. M. Coussens, "Paradoxical roles of the immune system during cancer development," Nature Reviews Cancer, vol. 6, no. 1, pp. 24-37, 2006.

[38] M. H. Andersen, B. S. Sørensen, M. K. Brimnes, I. M. Svane, J. C. Becker, and P. T. Straten, "Identification of heme oxygenase-1-specific regulatory $\mathrm{CD}^{+} \mathrm{T}$ cells in cancer 
patients," Journal of Clinical Investigation, vol. 119, no. 8, pp. 2245-2256, 2009.

[39] F. A. Wagener, H. E. van Beurden, J. W. von den Hoff, G. J. Adema, and C. G. Figdor, "The heme-heme oxygenase system: a molecular switch in wound healing," Blood, vol. 102, no. 2, pp. 521-528, 2003.

[40] M. P. Soares, I. Marguti, A. Cunha, and R. Larsen, "Immunoregulatory effects of $\mathrm{HO}-1$ : how does it work?" Current Opinion in Pharmacology, vol. 9, no. 4, pp. 482-489, 2009.

[41] G. C. Prendergast, "Immune escape as a fundamental trait of cancer: focus on IDO," Oncogene, vol. 27, no. 28, pp. 38893900, 2008.

[42] C. Uyttenhove, L. Pilotte, I. Théate et al., "Evidence for a tumoral immune resistance mechanism based on tryptophan degradation by indoleamine 2,3-dioxygenase," Nature Medicine, vol. 9, no. 10, pp. 1269-1274, 2003.

[43] B. Baban, P. R. Chandler, M. D. Sharma et al., "IDO activates regulatory $\mathrm{T}$ cells and blocks their conversion into Th17-like T cells," Journal of Immunology, vol. 183, no. 4, pp. 24752483, 2009.

[44] P. Thebault, T. Condamine, M. Heslan et al., "Role of IFN $\gamma$ in allograft tolerance mediated by $\mathrm{CD} 4{ }^{+} \mathrm{CD} 25^{+}$regulatory T cells by induction of IDO in endothelial cells," American Journal of Transplantation, vol. 7, no. 11, pp. 2472-2482, 2007.

[45] D.-Y. Hou, A. J. Muller, M. D. Sharma et al., "Inhibition of indoleamine 2,3-dioxygenase in dendritic cells by stereoisomers of 1-methyl-tryptophan correlates with antitumor responses," Cancer Research, vol. 67, no. 2, pp. 792-801, 2007.

[46] R. B. Sorensen, P. T. Straten, and M. H. Andersen, "Comment on "reduced cytotoxic function of effector $\mathrm{CD} 8^{+} \mathrm{T}$ cells is responsible for indoleamine 2,3-dioxygenase-dependent immune suppression"', Journal of Immunology, vol. 183, no. 10, p. 6040, 2009.

[47] R. J. North, "Cyclophosphamide-facilitated adoptive immunotherapy of an established tumor depends on elimination of tumor-induced suppressor T cells," Journal of Experimental Medicine, vol. 155, no. 4, pp. 1063-1074, 1982.

[48] D. Berd, H. C. Maguire Jr., and M. J. Mastrangelo, "Induction of cell-mediated immunity to autologous melanoma cells and regression of metastases after treatment with a melanoma cell vaccine preceded by cyclophosphamide," Cancer Research, vol. 46, no. 5, pp. 2572-2577, 1986.

[49] S. Brode and A. Cooke, "Immune-potentiating effects of the chemotherapeutic drug cyclophosphamide," Critical Reviews in Immunology, vol. 28, no. 2, pp. 109-126, 2008.

[50] M. Vukmanovic-Stejic, E. Agius, N. Booth et al., "The kinetics of $\mathrm{CD} 4^{+} \mathrm{Foxp}^{+} \mathrm{T}$ cell accumulation during a human cutaneous antigen-specific memory response in vivo," Journal of Clinical Investigation, vol. 118, no. 11, pp. 3639-3650, 2008.

[51] L. Zhang, K. Dermawan, M. Jin et al., "Differential impairment of regulatory $\mathrm{T}$ cells rather than effector $\mathrm{T}$ cells by paclitaxel-based chemotherapy," Clinical Immunology, vol. 129, no. 2, pp. 219-229, 2008.

[52] O. T. M. Chan and L.-X. Yang, "The immunological effects of taxanes," Cancer Immunology Immunotherapy, vol. 49, no. 4-5, pp. 181-185, 2000.

[53] X. Wu, Q.-M. Feng, Y. Wang, J. Shi, H.-L. Ge, and W. Di, “The immunologic aspects in advanced ovarian cancer patients treated with paclitaxel and carboplatin chemotherapy," Cancer Immunology, Immunotherapy, vol. 59, pp. 279-291, 2010.
[54] M. Tongu, N. Harashima, T. Yamada, T. Harada, and M. Harada, "Immunogenic chemotherapy with cyclophosphamide and doxorubicin against established murine carcinoma," Cancer Immunology, Immunotherapy, vol. 59, pp. 769-777, 2010.

[55] M. Vukmanovic-Stejic, Y. Zhang, J. E. Cook et al., "Human $\mathrm{CD} 4{ }^{+} \mathrm{CD} 25^{\text {hi }}$ Foxp $3^{+}$regulatory $\mathrm{T}$ cells are derived by rapid turnover of memory populations in vivo," Journal of Clinical Investigation, vol. 116, no. 9, pp. 2423-2433, 2006.

[56] E. Suzuki, V. Kapoor, A. S. Jassar, L. R. Kaiser, and S. M. Albelda, "Gemcitabine selectively eliminates splenic $\mathrm{Gr}-1^{+} / \mathrm{CD} 11 \mathrm{~b}^{+}$myeloid suppressor cells in tumor-bearing animals and enhances antitumor immune activity," Clinical Cancer Research, vol. 11, no. 18, pp. 6713-6721, 2005.

[57] V. Bronte, T. Kasic, G. Gri et al., "Boosting antitumor responses of $\mathrm{T}$ lymphocytes infiltrating human prostate cancers," Journal of Experimental Medicine, vol. 201, no. 8, pp. 1257-1268, 2005.

[58] J. E. Talmadge, K. C. Hood, L. C. Zobel, L. R. Shafer, M. Coles, and B. Toth, "Chemoprevention by cyclooxygenase2 inhibition reduces immature myeloid suppressor cell expansion," International Immunopharmacology, vol. 7, no. 2, pp. 140-151, 2007.

[59] P. Sinha, V. K. Clements, A. M. Fulton, and S. OstrandRosenberg, "Prostaglandin E2 promotes tumor progression by inducing myeloid-derived suppressor cells," Cancer Research, vol. 67, no. 9, pp. 4507-4513, 2007.

[60] S. Nagaraj, J.-I. Youn, H. Weber, et al., "Anti-inflammatory triterpenoid blocks immune suppressive function of MDSCs and improves immune response in cancer," Clinical Cancer Research, vol. 16, no. 6, pp. 1812-1823, 2010.

[61] S. Nagaraj, A. G. Schrum, H. I. Cho, E. Celis, and D. I. Gabrilovich, "Mechanism of $\mathrm{T}$ cell tolerance induced by myeloid-derived suppressor cells," Journal of Immunology, vol. 184, no. 6, pp. 3106-3116, 2010.

[62] I. Poschke, D Mougiakakos, J. Hansson, G. Masucci, and R. Kiessling, "Immature immunosuppressive CD14+ HLADRlow cells inmelanoma patients are STAT3hi and overexpress CD80, CD83 and DC-Sign," Cancer Research. In press.

[63] D. Laheru, E. Lutz, J. Burke et al., "Allogeneic granulocyte macrophage colony-stimulating factor-secreting tumor immunotherapy alone or in sequence with cyclophosphamide for metastatic pancreatic cancer: a pilot study of safety, feasibility, and immune activation," Clinical Cancer Research, vol. 14, no. 5, pp. 1455-1463, 2008.

[64] M. E. Dudley and S. A. Rosenberg, "Adoptive cell transfer therapy," Seminars in Oncology, vol. 34, no. 6, pp. 524-531, 2007.

[65] M. Cobbold, N. Khan, B. Pourgheysari et al., "Adoptive transfer of cytomegalovirus-specific CTL to stem cell transplant patients after selection by HLA-peptide tetramers," Journal of Experimental Medicine, vol. 202, no. 3, pp. 379-386, 2005.

[66] R. A. Morgan, M. E. Dudley, J. R. Wunderlich et al., "Cancer regression in patients after transfer of genetically engineered lymphocytes," Science, vol. 314, no. 5796, pp. 126-129, 2006.

[67] A. P. Rapoport, E. A. Stadtmauer, N. Aqui et al., "Restoration of immunity in lymphopenic individuals with cancer by vaccination and adoptive T-cell transfer," Nature Medicine, vol. 11, no. 11, pp. 1230-1237, 2005.

[68] E. Suzuki, J. Sun, V. Kapoor, A. S. Jassar, and S. M. Albelda, "Gemcitabine has significant immunomodulatory activity in murine tumor models independent of its cytotoxic effects," Cancer Biology and Therapy, vol. 6, no. 6, pp. 880-885, 2007. 
[69] V. Radojcic, K. B. Bezak, M. Skarica et al., "Cyclophosphamide resets dendritic cell homeostasis and enhances antitumor immunity through effects that extend beyond regulatory T cell elimination," Cancer Immunology, Immunotherapy, vol. 59, no. 1, pp. 137-148, 2010.

[70] H. Tanaka, H. Matsushima, N. Mizumoto, and A. Takashima, "Classification of chemotherapeutic agents based on their differential in vitro effects on dendritic cells," Cancer Research, vol. 69, no. 17, pp. 6978-6986, 2009.

[71] H. Tanaka, H. Matsushima, A. Nishibu, B. E. Clausen, and A. Takashima, "Dual therapeutic efficacy of vinblastine as a unique chemotherapeutic agent capable of inducing dendritic cell maturation," Cancer Research, vol. 69, no. 17, pp. 6987-6994, 2009.

[72] G. V. Shurin, I. L. Tourkova, R. Kaneno, and M. R. Shurin, "Chemotherapeutic agents in noncytotoxic concentrations increase antigen presentation by dendritic cells via an IL-12dependent mechanism," Journal of Immunology, vol. 183, no. 1, pp. 137-144, 2009.

[73] M. Obeid, A. Tesniere, F. Ghiringhelli et al., "Calreticulin exposure dictates the immunogenicity of cancer cell death," Nature Medicine, vol. 13, no. 1, pp. 54-61, 2007.

[74] K. M. Livesey, D. Tang, H. J. Zeh, and M. T. Lotze, "Autophagy inhibition in combination cancer treatment," Current Opinion in Investigational Drugs, vol. 10, no. 12, pp. 1269-1279, 2009.

[75] J. E. Ellerman, C. K. Brown, M. de Vera et al., "Masquerader: high mobility group box-1 and cancer," Clinical Cancer Research, vol. 13, no. 10, pp. 2836-2848, 2007.

[76] H. Jensen, L. Andresen, K. A. Hansen, and S. Skov, "Cellsurface expression of Hsp70 on hematopoietic cancer cells after inhibition of HDAC activity," Journal of Leukocyte Biology, vol. 86, no. 4, pp. 923-932, 2009.

[77] R. Spisek, A. Charalambous, A. Mazumder, D. H. Vesole, S. Jagannath, and M. V. Dhodapkar, "Bortezomib enhances dendritic cell (DC)-mediated induction of immunity to human myeloma via exposure of cell surface heat shock protein 90 on dying tumor cells: therapeutic implications," Blood, vol. 109, no. 11, pp. 4839-4845, 2007.

[78] E. S. Bergmann-Leitner and S. I. Abrams, "Treatment of human colon carcinoma cell lines with anti-neoplastic agents enhances their lytic sensitivity to antigen-specific CD8+ cytotoxic T lymphocytes," Cancer Immunology, Immunotherapy, vol. 50, no. 9, pp. 445-455, 2001.

[79] X. X. Wu, Y. Zeng, X. H. Jin, and Y. Kakehi, "Enhanced susceptibility of adriamycin-treated human renal cell carcinoma cells to lysis by peripheral blood lymphocytes and tumor infiltrating lymphocytes," Oncology Reports, vol. 18, no. 2, pp. 353-359, 2007.

[80] R. Ramakrishnan, D. Assudani, S. Nagaraj, et al., "Chemotherapy enhances tumor cell susceptibility to CTL-mediated killing during cancer immunotherapy in mice," Journal of Clinical Investigation, vol. 120, no. 4, pp. 1111-1124, 2010

[81] K. Veugelers, B. Motyka, I. S. Goping, I. Shostak, T. Sawchuk, and R. C. Bleackley, "Granule-mediated killing by granzyme $B$ and perforin requires a mannose 6-phosphate receptor and is augmented by cell surface heparan sulfate," Molecular Biology of the Cell, vol. 17, no. 2, pp. 623-633, 2006.

[82] J. L. Frazier, J. E. Han, M. Lim, and A. Olivi, "Immunotherapy combined with chemotherapy in the treatment of tumors," Neurosurgery Clinics of North America, vol. 21, no. 1, pp. 187194, 2010.
[83] N. M. Haynes, R. G. van der Most, R. A. Lake, and M. J. Smyth, "Immunogenic anti-cancer chemotherapy as an emerging concept," Current Opinion in Immunology, vol. 20, no. 5, pp. 545-557, 2008.

[84] G. Makin and J. A. Hickman, "Apoptosis and cancer chemotherapy," Cell and Tissue Research, vol. 301, no. 1, pp. 143-152, 2000.

[85] K. M. Redmond, T. R. Wilson, P. G. Johnston, and D. B. Longley, "Resistance mechanisms to cancer chemotherapy," Frontiers in Bioscience, vol. 13, no. 13, pp. 5138-5154, 2008.

[86] D. B. Longley and P. G. Johnston, "Molecular mechanisms of drug resistance," Journal of Pathology, vol. 205, no. 2, pp. 275-292, 2005.

[87] S. Shangary and D. E. Johnson, "Recent advances in the development of anticancer agents targeting cell death inhibitors in the Bcl-2 protein family," Leukemia, vol. 17, no. 8, pp. 1470-1481, 2003.

[88] B. Rochat, J. M. Morsman, G. I. Murray, W. D. Figg, and H. L. Mcleod, "Human CYP1B1 and anticancer agent metabolism: mechanism for tumor-specific drug inactivation?” Journal of Pharmacology and Experimental Therapeutics, vol. 296, no. 2, pp. 537-541, 2001.

[89] P. Borst, R. Evers, M. Kool, and J. Wijnholds, "A family of drug transporters: the multidrug resistance-associated proteins," Journal of the National Cancer Institute, vol. 92, no. 16, pp. 1295-1302, 2000.

[90] R. B. Sørensen, O. J. Nielsen, P. T. Straten, and M. H. Andersen, "Functional capacity of Mcl-1-specific cytotoxic T-cells," Leukemia, vol. 20, no. 8, pp. 1457-1458, 2006.

[91] M. H. Andersen, L. $\varnothing$ Pedersen, J. C. Becker, and P. T. Straten, "Identification of a cytotoxic T lymphocyte response to the apoptose inhibitor protein Survivin in cancer patients," Cancer Research, vol. 61, pp. 869-872, 2001.

[92] M. H. Andersen, L. Ø. Pedersen, B. Capeller, E.-B. Bröcker, J. C. Becker, and P. T. Straten, "Spontaneous cytotoxic T-cell responses against survivin-derived MHC class I-restricted Tcell epitopes in situ as well as ex vivo in cancer patients," Cancer Research, vol. 61, no. 16, pp. 5964-5968, 2001.

[93] M. H. Andersen, S. Reker, J. C. Becker, and P. T. Straten, "The melanoma inhibitor of apoptosis protein: a target for spontaneous cytotoxic T cell responses," Journal of Investigative Dermatology, vol. 122, no. 2, pp. 392-399, 2004.

[94] M. H. Andersen, I. M. Svane, P. Kvistborg et al., "Immunogenicity of Bcl-2 in patients with cancer," Blood, vol. 105, no. 2, pp. 728-734, 2005.

[95] M. H. Andersen, S. Reker, P. Kvistborg, J. C. Becker, and P. T. Straten, "Spontaneous immunity against Bcl-xL in cancer patients," Journal of Immunology, vol. 175, no. 4, pp. 27092714, 2005.

[96] A. J. Munro, "Bystander effects and their implications for clinical radiotherapy," Journal of Radiological Protection, vol. 29, no. 2A, pp. A133-A142, 2009.

[97] E. Jäger, M. Ringhoffer, M. Altmannsberger et al., "Immunoselection in vivo: independent loss of MHC class I and melanocyte differentiation antigen expression in metastatic melanoma," International Journal of Cancer, vol. 71, no. 2, pp. 142-147, 1997.

[98] B. Seliger, U. Ritz, and S. Ferrone, "Molecular mechanisms of HLA class I antigen abnormalities following viral infection and transformation," International Journal of Cancer, vol. 118, no. 1, pp. 129-138, 2006.

[99] A. Tandle, D. G. Blazer III, and S. K. Libutti, "Antiangiogenic gene therapy of cancer: recent developments," Journal of Translational Medicine, vol. 2, article 22, 2004. 
[100] H. Ishizaki, T. Tsunoda, S. Wada, M. Yamauchi, M. Shibuya, and H. Tahara, "Inhibition of tumor growth with antiangiogenic cancer vaccine using epitope peptides derived from human vascular endothelial growth factor receptor 1," Clinical Cancer Research, vol. 12, no. 19, pp. 5841-5849, 2006.

[101] S. Dias, S. V. Shmelkov, G. Lam, and S. Rafii, "VEGF 165 promotes survival of leukemic cells by Hsp90-mediated induction of Bcl-2 expression and apoptosis inhibition," Blood, vol. 99, no. 7, pp. 2532-2540, 2002.

[102] R. Xiang, N. Mizutani, Y. Luo et al., "A DNA vaccine targeting survivin combines apoptosis with suppression of angiogenesis in lung tumor eradication," Cancer Research, vol. 65, no. 2, pp. 553-561, 2005.

[103] K. Otto, M. H. Andersen, and A. A. Eggert, "Therapyinduced $\mathrm{T}$ cell responses against the universal tumor antigen survivin," Vaccine, vol. 23, pp. 884-889, 2004.

[104] C. L. Slingluff Jr., G. R. Petroni, K. A. Chianese-Bullock et al., "Immunologic and clinical outcomes of a randomized phase II trial of two multipeptide vaccines for melanoma in the adjuvant setting," Clinical Cancer Research, vol. 13, no. 21, pp. 6386-6395, 2007.

[105] R. B. Sørensen, I. M. Svane, P. T. Straten, and M. H. Andersen, "A survivin specific T-cell clone from a breast cancer patient display universal tumor cell lysis," Cancer Biology and Therapy, vol. 7, no. 12, pp. 1885-1887, 2008.

[106] R. B. Sørensen, M. Faurschou, L. Troelsen et al., "Melanoma inhibitor of apoptosis protein (ML-IAP) specific cytotoxic T lymphocytes cross-react with an epitope from the autoantigen SS56," Journal of Investigative Dermatology, vol. 129, no. 8, pp. 1992-1999, 2009.

[107] M. P. Patel, A. Masood, P. S. Patel, and A. A. Chanan-Khan, "Targeting the Bcl-2," Current Opinion in Oncology, vol. 21, pp. 516-523, 2009.

[108] H. Yamamoto, C. Y. Ngan, and M. Monden, "Cancer cells survive with survivin,” Cancer Science, vol. 99, no. 9, pp. 1709-1714, 2008.

[109] J. A. Shafer, C. R. Cruz, A. M. Leen et al., "Antigen-specific cytotoxic T lymphocytes can target chemoresistant sidepopulation tumor cells in Hodgkin lymphoma," Leukemia and Lymphoma, vol. 51, no. 5, pp. 870-880, 2010.

[110] K. Tanaka, S. Iwamoto, G. Gon, T. Nohara, M. Iwamoto, and N. Tanigawa, "Expression of survivin and its relationship to loss of apoptosis in breast carcinomas," Clinical Cancer Research, vol. 6, no. 1, pp. 127-134, 2000.

[111] E. A. Clark, T. R. Golub, E. S. Lander, and R. O. Hynes, "Genomic analysis of metastasis reveals an essential role for RhoC," Nature, vol. 406, no. 6795, pp. 532-535, 2000. 


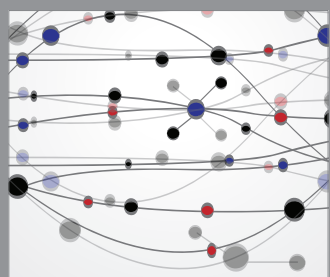

The Scientific World Journal
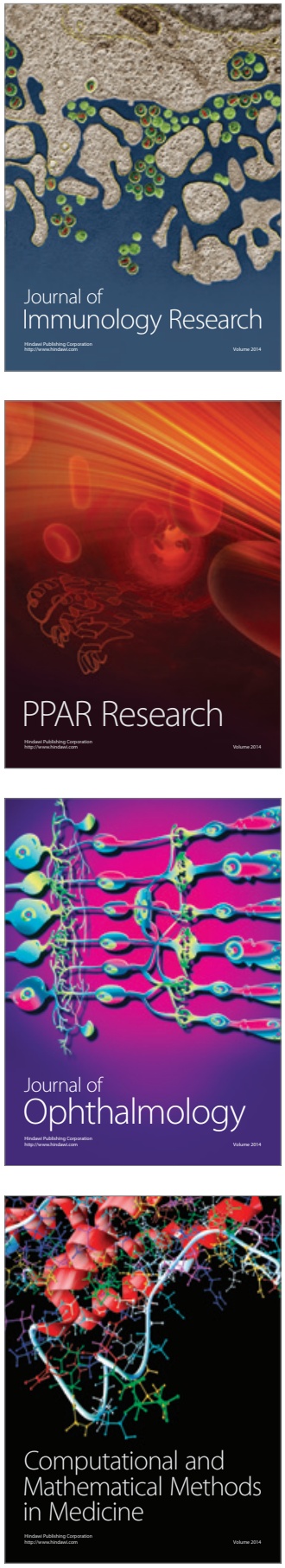

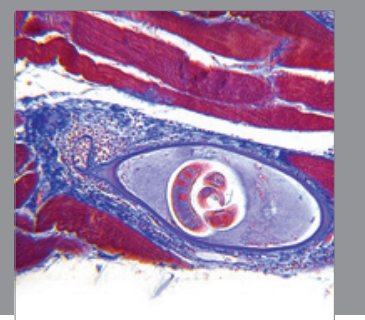

Gastroenterology

Research and Practice
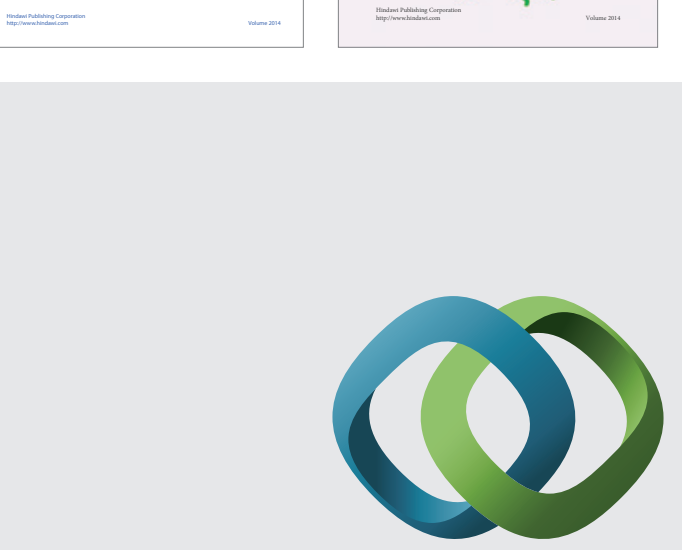

\section{Hindawi}

Submit your manuscripts at

http://www.hindawi.com
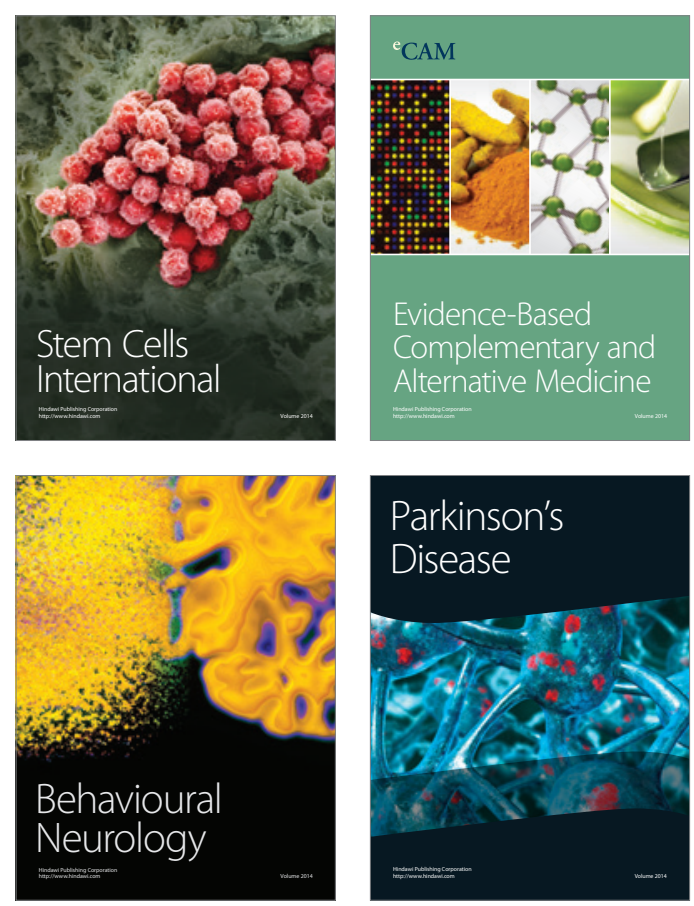

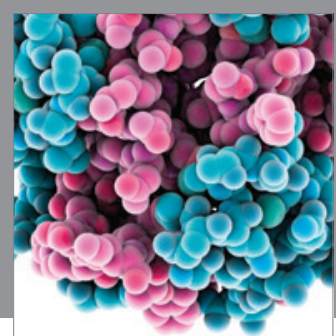

Journal of
Diabetes Research

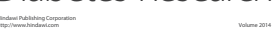

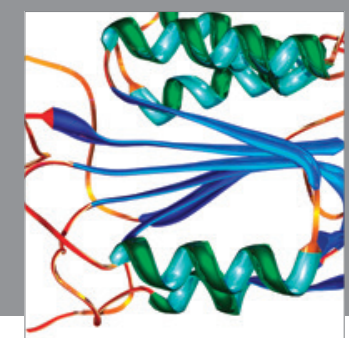

Disease Markers
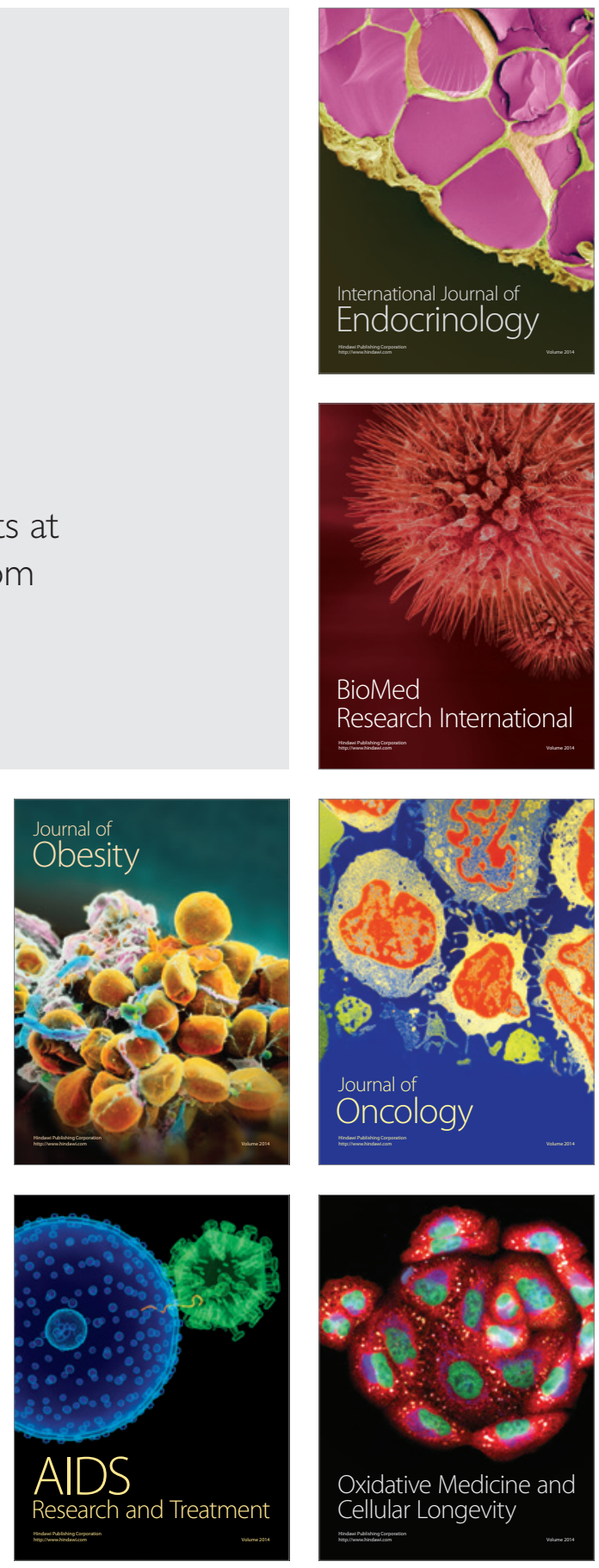